\title{
Highly adaptable gain-switched fiber laser with improved efficiency
}

\author{
VID AGREŽ AND ROK PETKOVŠEK \\ University of Ljubljana, Faculty of Mechanical Engineering, Aškerčeva 6, SI-1000 Ljubljana, Slovenia \\ *vid.agrez@fs.uni-lj.si
}

\begin{abstract}
A highly adaptable fiber laser with pulse-on-demand and precision pulse-duration tuning is presented. It is based on a compact optical design combining the gain-switching technique with the all-fiber master oscillator and pump-recovery amplifier architecture. The approach of laser-pulse stability control by compensation pumping and pulse-duration control by changing the pump wavelength are introduced. In order to prove the concept, a laser setup capable of producing laser pulses with an average power of up to $30 \mathrm{~W}$ and a peak power of approximately $1 \mathrm{~kW}$ at an improved efficiency and an arbitrary repetition rate is presented.
\end{abstract}

(C) 2019 Optical Society of America under the terms of the OSA Open Access Publishing Agreement

\section{Introduction}

Today's laser-based manufacturing processes and some laser treatments in medicine require highly flexible production tools to make more advanced patterns and structures, potentially at high speed. Such processes could benefit from highly adaptable laser systems that can produce an on-demand pulse and also control other pulse parameters in combination with a high-speed scanning system based on a polygon or resonant scanner. Such laser systems are usually complex because they must include additional optical elements, i.e., modulators and their drivers. For several applications, such as micromachining, laser direct color marking, laser transfer printing and even some special medical treatment (like selective photocoagulation), laser pulses with a duration of several $10 \mathrm{~s}$ of ns at relatively low peak power (the $\mathrm{kW}$ range or even below) are required. Here, an example of an efficient solution is the gain-switched laser [1], which can effectively maintain pulse parameters across a broad range of repetition rates [2]. It was shown that with the right optimization of the laser's physical parameters, short nanosecond pulses can be achieved [3]. Such pulses typically have a peak power that is 10 times larger than the absorbed peak pump power.

An unwanted characteristic of gain-switched lasers is the laser pulse's dependence on the physical parameters of the oscillator (length and pump absorption), which for the given laser system fixes the output pulse duration to a peak power ratio [4]. For example, short duration pulses (generated in the gain-switched mode) require a short resonator length, which leads to a decrease in the overall efficiency (typically less than 30\%). This can be addressed by adding an additional active fiber that is pumped by a residual (unabsorbed) pump from the main oscillator [5]. Such a fiber-laser architecture maintains the simplicity and compactness of the gain-switched laser and can be applied to lasers operating in applications where robustness and low maintenance are a requirement, pulsed operation at exotic wavelengths, where there is lack of the appropriate active optical elements for pulse generation, or as a high-brightness pump source for core pumping applications.

The gain-switched technique was mainly developed with ytterbium- or thulium-doped active fibers. For example, to the best of our knowledge, the shortest pulses achieved to date from $\mathrm{Yb}$-doped fiber lasers were $28 \mathrm{~ns}$ [3], with even shorter pulses (17 ns) being produced from a core-pumped Tm-doped fiber [6] by a 1550-nm pulsed pump source. Also, the clad pumping of Tm-doped fibers can be realized [7], enabled by efficient pumping at $790 \mathrm{~nm}$, which is not susceptible to photo darkening and can achieve up to 64\% slope efficiency [8]. By combining the technique with thulium- and holmium-doped active fibers, a spectral region 
of up to $2.1 \mu \mathrm{m}$ was achieved [9], while an erbium-doped fluoride fiber [10] produced pulses at $2.8 \mu \mathrm{m}$.

In this paper we show an advanced, all-fiber, gain-switched, ytterbium-doped fiber laser that is capable of operating at arbitrary repetition rates from a single pulse to approximately 1 $\mathrm{MHz}$ using a technique based on gain control to maintain the laser always slightly below the operation threshold. This allows the repetition rate of the laser to be changed instantly and real pulse-on-demand can be achieved. Furthermore, the laser is capable of changing its pulse duration while preserving the peak power while operating at $1030 \mathrm{~nm}$, which is otherwise hard to obtain with gain-switched lasers based only on the oscillator. This is achieved by introducing another tuning parameter: an effective pump absorption that allows for pump power distribution control between the oscillator and the amplifier, and thus changing of the pulse duration while maintaining the total efficiency of the laser.

The biggest advantage of the proposed gain-switched laser system compared to the gainswitched oscillator with a separate added amplifier is its low complexity. The proposed laser is much simpler and more cost effective to build, as it eliminates the need for a feed-through combiner and uses only one pump system based on pump laser diodes for providing a pulseon-demand and pulse-tuning functionality. In addition, the existing solutions of gain-switched lasers have the peak power changed when setting the pulse duration. For short laser pulses a short oscillator length is required, which then lowers the efficiency. The efficiency is also lowered when operating in pulse-on-demand mode. In comparison, the pump-recovery amplifier together with the compensation pumping presented in this paper allows for high efficiency at a constant laser-pulse peak power when producing pulses on demand and changing the pulse duration.

The approach presented in this paper for a laser pulse-on-demand operating mode and pulse tuning is not limited to ytterbium-doped fibers, but can benefit the design and applications of gain-switched fiber lasers based on different kinds of active fibers covering a currently very interesting range of wavelengths that are longer than $2 \mu \mathrm{m}$.

\section{Theory}

The gain-switched operation of a pulsed-laser system is based on the high-speed modulation of the pump power that produces relaxation oscillations of the laser output power. By switching off the pump laser diodes fast enough, only the first relaxation spike can be selected. This spike can be formed in a controllable manner and represents the output laser pulse. The dynamics of gain switching were already described in $[4,11,12]$ and a good approximation connecting the laser pulse duration $t_{0}$ to the laser parameters can be derived from rate equations describing the laser photon and inversion populations:

$$
t_{0}=\frac{2}{\pi} \sqrt{\frac{n A h}{\Gamma \sigma_{e} \lambda_{p}}} \sqrt{\frac{L_{p}+L_{d}}{P_{p}\left(1-\exp \left(-0.23 \alpha L_{d}\right)\right)}} .
$$

Equation (1) is written in such a way that the important parameters influencing the pulse duration are grouped together in the second square-root term: $L_{\mathrm{p}}$ is the passive fiber length, $L_{\mathrm{d}}$ is the doped fiber length, $P_{\mathrm{p}}$ is the pump peak power and $\alpha$ is the absorption coefficient. The other parameters are as follows: $n$ is the refractive index of the fiber, $A$ is the doped area cross-section, $h$ is Planck's constant, $\Gamma$ is the overlap integral between the signal and the fiber core, $\sigma_{\mathrm{e}}$ is the emission cross-section and $\lambda_{\mathrm{p}}$ is the pump wavelength.

\section{Pulse-duration tuning}

As can be seen from Eq. (1), most of the parameters that affect the laser pulse must be predefined when designing the laser system. The only free parameter left is the absorbed pump power, which depends on the available power of the pump diodes and the absorption coefficient of the pump light. In order to keep the laser peak power constant, only the latter is 
available for the tuning and must be used with the proposed gain-switched oscillator with the regenerative amplifier setup to have an effect. Selecting the narrow ytterbium absorption peak makes it possible to tune the effective absorption coefficient in the laser by the factor 4 , as can be seen in Fig. 1(a).

This allows for maximum pulse-duration tuning up to a factor of 2 (Fig. 1(b)). The results of the model shown are the calculated ratio of the achievable pulse duration to the minimum pulse duration at the corresponding length of the doped fiber in the oscillator. We must also take into account the reduced amount of power deposited in the oscillator, so the optimal doped oscillator length to produce stable gain-switched pulses is between 0.2 and $0.6 \mathrm{~m}$ (Fig. $1(\mathrm{c}))$.
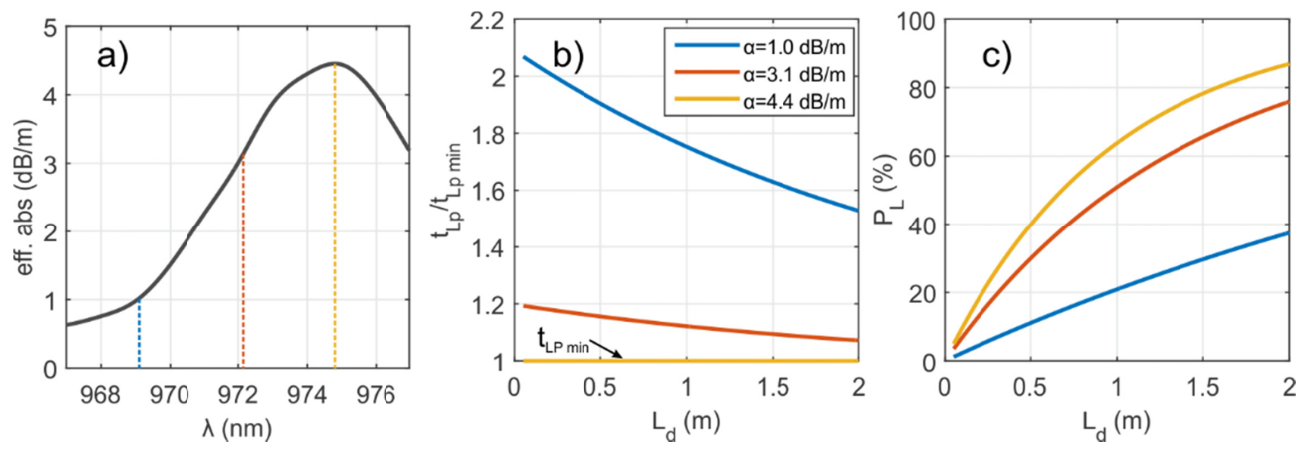

Fig. 1. Results of the analytical model with respect to the effective absorption coefficient. a) Change of the effective absorption coefficient with the pump wavelength. b) Increase of the pulse duration in comparison to the minimal pulse duration at different oscillator fiber lengths. c) Ratio of the oscillator output power to the maximum output power.

\section{Pulse on demand}

The gain-switched laser architecture based on an all-fiber design, including the pump recovery amplifier that allows for laser-pulse-duration tuning and a higher laser efficiency, is further analyzed with regards to operating at different repetition rates, with the aim of developing a pulse-on-demand gain-switched laser. To describe the system's dependency on the repetition rate, the operation thresholds for both the laser oscillator and the amplifier are derived.

For the oscillator the threshold population $\left(N_{2 \mathrm{~L}}\right)$ connected to the resonator losses and as a consequence to the photon lifetime $\left(\tau_{\mathrm{f}}\right)$ in the oscillator can be derived from the rate equations [2]:

$$
N_{2 \mathrm{~L}}=\frac{V}{\Gamma \sigma_{e} c \tau_{f}}+N_{1}=\frac{-\ln \left(R_{1} R_{2}\right) A}{2 \Gamma \sigma_{e}} \frac{L_{d}}{L_{d}+L_{p}}+N_{1}
$$

that can be further normalized to all the active ions in the oscillator $\left(N_{\mathrm{d}}=n_{\mathrm{d}} V\right)$ :

$$
r_{\mathrm{L}}=\frac{N_{2 \mathrm{~L}}}{N_{\mathrm{d}}}=\frac{-\ln \left(R_{1} R_{2}\right)}{2 \Gamma \sigma\left(\mathrm{L}_{d}+L_{p}\right) n_{d}}+\frac{N_{1}}{N_{\mathrm{d}}} .
$$

Because the ytterbium-doped active fibers are a quasi-four-level gain medium the thermal occupancy of the lower laser level $\left(N_{1}\right)$ cannot be neglected. It can be expressed as:

$$
r_{\mathrm{T}}=\frac{N_{1}}{N_{\mathrm{d}}}=\exp \left(-\frac{E_{2}-h c / \lambda_{\mathrm{s}}}{k_{\mathrm{B}} T}\right)
$$


where $E_{2}$ is the energy of the upper laser level $\left(E_{2}=1.273 \mathrm{eV}\right.$ for an ytterbium-doped silica fiber), $\lambda_{\mathrm{s}}$ is the signal wavelength $\left(\lambda_{\mathrm{s}}=1030 \mathrm{~nm}\right)$ and $T$ is the temperature. This value also represents the amplifier threshold.

The system is pumped with the photon rate $W$, corresponding to the absorbed pump light $\left(P_{\text {abs }}\right)$ in the oscillator and the amplifier:

$$
W=\frac{\lambda_{\mathrm{p}} P_{\mathrm{abs}}}{h c_{0}} .
$$

As the active fiber volume of the oscillator and the amplifier are different, and as a consequence have a different number of active ions, the average upper-level population of the amplifier approaches the threshold more slowly than in the oscillator. This behavior is shown in Fig. 2 for the setup described in the experimental section. When pumped with a pumppulse power of $100 \mathrm{~W}$ the oscillator reaches the operation threshold $r_{\mathrm{T}}=11 \%$ at $1.4 \mu \mathrm{s}$. At this time the average exited-ion population in the amplifier is still well under the threshold of $1.6 \%$, which means that the amplifier does not contribute to the laser-pulse amplification. The described example is the case of a cold start to the laser's operation. A similar situation occurs when operating at repetition rates comparable with or lower than the $1 \mathrm{kHz}$ that corresponds to the spontaneous emission significantly depleting the upper laser level. By increasing the repetition rate, the difference in the pumped condition before the laser-pulse generation between the oscillator and the amplifier becomes smaller and the amplifier starts to amplify the generated laser pulses from the oscillator. The smaller difference in the pumped condition is due to the spontaneous emission not having the time to significantly deplete the upper laser level of the oscillator and the amplifier. By adapting the pump system to hold the whole pumped laser slightly below the threshold, the influence of the spontaneous emission can be mitigated.

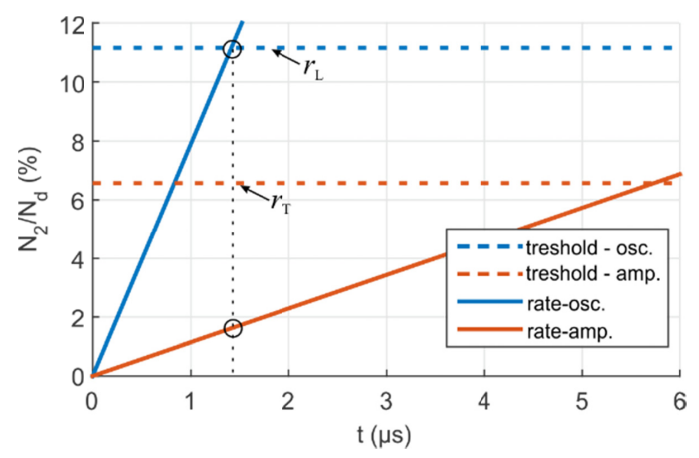

Fig. 2. Comparison of the upper laser level build up in the laser oscillator and the amplifier, which are arranged and pumped as described in the section Experimental with a pump-pulse power of $100 \mathrm{~W}$. It is clear that when (at $1.4 \mu \mathrm{s}$ ) the oscillator reaches the operating threshold the average exited-ion population in the amplifier is still well below the threshold of $1.6 \%$.

\section{Experimental}

The gain-switched laser architecture based on an all-fiber design, including the pumprecovery amplifier, is shown in Fig. 3. The laser system was based on a commercial, active, ytterbium-doped LMA fiber with a core diameter of $10 \mu \mathrm{m}$ and a cladding diameter of 125 $\mu \mathrm{m}$ using a section of length $0.5 \mathrm{~m}$ for the oscillator and $4 \mathrm{~m}$ for the amplifier. The oscillator was formed by fiber Bragg gratings with a high reflectivity of $R_{1}=99 \%$ (HR FBG) and a low reflectivity of $R_{2}=5 \%$ (OC FBG). The active fiber of the pump-recovery amplifier was spliced directly to the passive fiber of the low-reflectivity FBG and the angle cleaved at the output end to prevent back reflection. The length of the oscillator was chosen through the simulation as it offers the best trade off between the pulse length and the absorbed power. 
Tuning the laser-pulse duration was accomplished by the pump power transfer between the oscillator Fig. 3(b) and the amplifier Fig. 3(c) through the wavelength control of the pump light. The system has a single control unit Fig. 3(a) including the laser-pulse stability control that is integrated directly into the laser front end with no need for additional optical components at the laser output, which further simplifies the design.

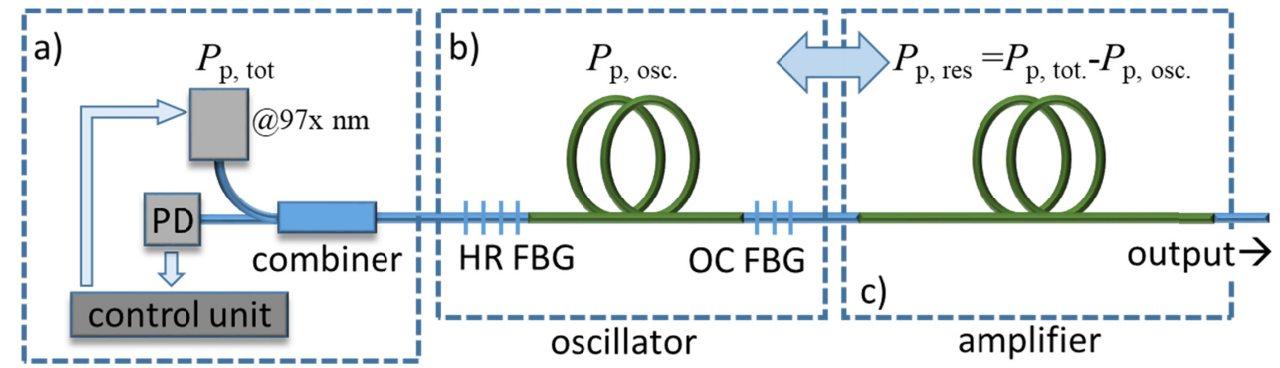

Fig. 3. Gain-switched laser architecture based on an all-fiber design including the pumprecovery amplifier. The pump power transfer between the oscillator and the amplifier through the wavelength control of the pump light enables laser-pulse-duration tuning and better efficiency. The stability of the laser pulses is enhanced through the photodiode (PD) and the feedback loop.

The measured output from the laser system for different pump conditions is shown in Fig. 4(a), where the D1 section shows damped oscillations and D2 the fully developed relaxation oscillations. The section D3 shows the system's response when the pump is switched off right after the laser pulse in the shape of the first relaxation oscillation (enlarged section shown in Fig. 4(c)). Figure 4(a) shows the pump and the laser trace in proportion, while in the insets Fig. 4(b) and Fig. 4(c) the transmitted pump power through the system is scaled by a factor of 6 to better show the transients caused by the rising and falling of the inversion population in the laser. Figure 4(b) clearly shows where the pump light should be switched off before the inversion population starts rising again. This approach to measuring the inversion population through the pump light absorption could also be used for the pump-pulse control.

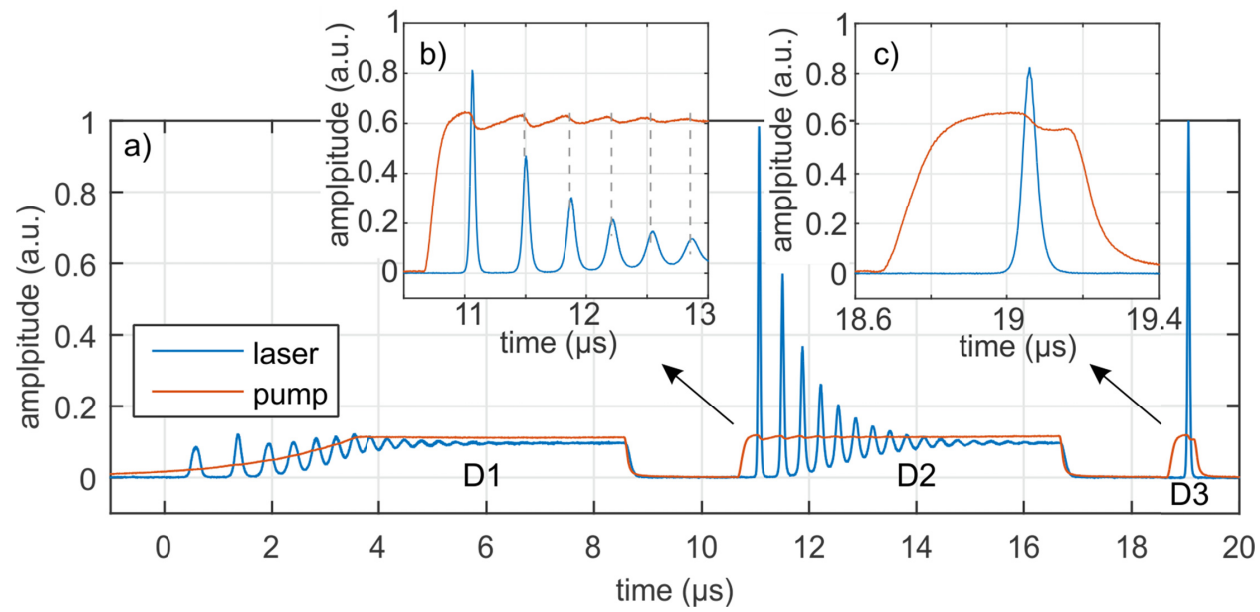

Fig. 4. Measured different pumping conditions of the laser system designed for producing gain-switched pulses are shown in a) where D1 shows the measured damped and D2 the undamped relaxation oscillations. The D3 pumping condition is for producing stable gainswitched pulses. 


\section{Results and discussion}

The presented laser system can operate with repetition rates up to $1 \mathrm{MHz}$, limited by the available control electronics and laser diode driver. Otherwise, the laser could produce stable pulses, as shown in Fig. 5, at a repetition rate of $500 \mathrm{kHz}$. The relation between the electric driving current pulse shape, the pump pulse with a peak power of $100 \mathrm{~W}$ and the generated laser pulse is shown in Fig. 5(a). To check the laser-pulse duration and the amplitude stability a stack of 250 successive pulses plotted together and fitted with the $\operatorname{sech}^{2}$ function is shown in Fig. 5(b), with good agreement between the measurements and the fit.
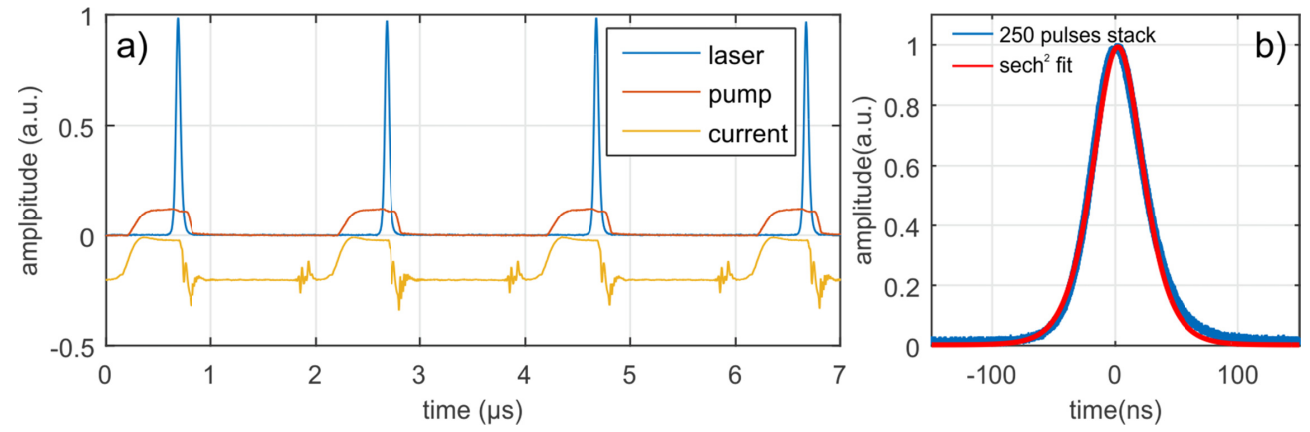

Fig. 5. a) Laser-pulse train at $500 \mathrm{kHz}$ shown together with the pump pulse and the driving current. A stack of 250 successive pulses plotted together is shown in b) and fitted with the $\operatorname{sech}^{2}$ function.

Furthermore, the tuning of the pulse duration, together with the peak power stability and the energy efficiency, was tested on the system at the same pump-pulse peak power of $100 \mathrm{~W}$. The results are shown in Fig. 6 for a repetition rate of $100 \mathrm{kHz}$. A laser-pulse duration tuning factor of 1.8 was achieved, which is in good agreement with the model result for the $0.5-\mathrm{m}$ fiber shown in Fig. 1(b). The peak power in this experiment was preserved as the power deposited in the whole system did not change significantly, in contrast to the setups containing only the oscillator. Another benefit of the concept is that the efficiency of the oscillator alone ranging from $10 \%$ to $30 \%$ was increased to a range from $65 \%$ to $75 \%$ for the oscillator with the pump-recovery amplifier. When using a gain-switched laser consisting of only the oscillator, the efficiency increases with the effective absorption coefficient due to more power being absorbed in the laser. Adding the pump-recovery amplifier increases the total efficiency, but shows a slight drop with the increased effective absorption coefficient due to less pumping power being available in the amplifier. With further optimization, like operation wavelength selection, this reduction in efficiency could be avoided. 

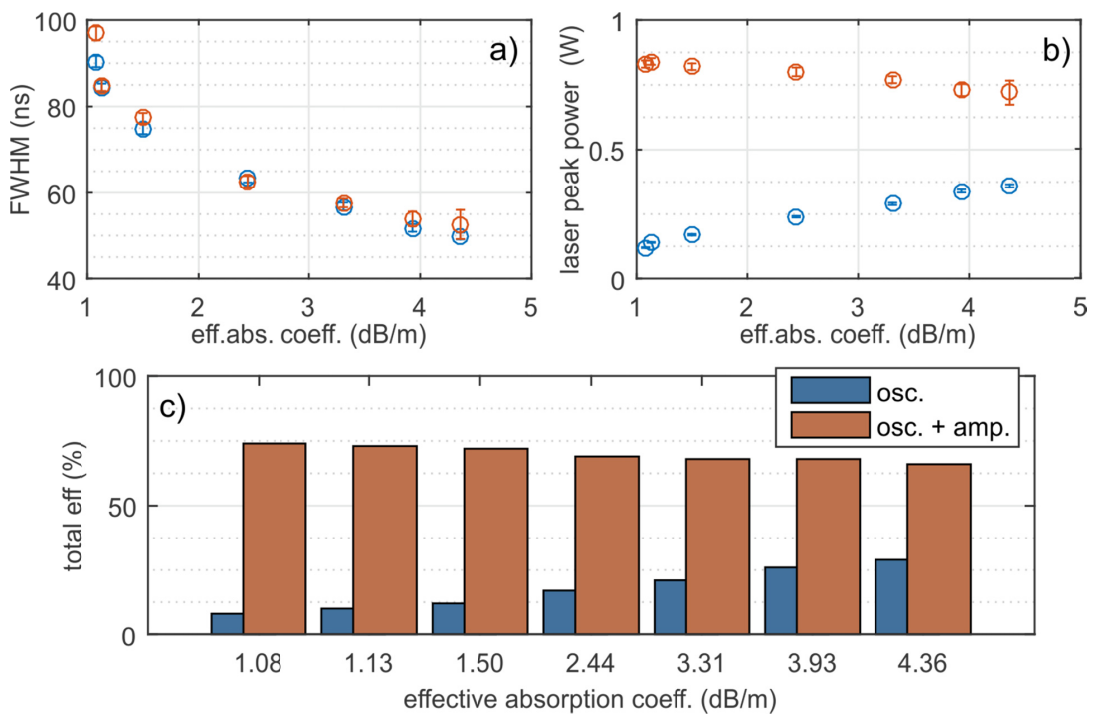

Fig. 6. Gain-switched, laser-output, pulse-parameter dependencies on the effective absorption coefficient are shown in a) for pulse FWHM, b) laser peak power and c) comparison of the efficiency between the two setups: oscillator only (10-30\%, blue bars) and the proposed system consisting of an oscillator and a pump-recovery amplifier ( $65-75 \%$, brown bars).

Using normal pumping with nearly square-shaped pump pulses and changing the time between the pulses from $2 \mu \mathrm{s}$ to $1 \mathrm{~ms}$, produced laser pulses that had a peak power reduced by a factor of 5 in the tested range (Fig. 7(b)). This happens despite the increase in the optimum pump-pulse duration (Fig. 7(a)), which is connected to the time required to produce the first relaxation oscillation from the laser oscillator.

The reduction in the peak power is connected to the uneven pump conditions along the laser optical path. As shown in Fig. 2, for a laser cold start, the oscillator produces a laser pulse before the amplifier reaches the threshold for amplification. The difference in the pump condition is related to the lifetime of the excited ions, which is around $0.9 \mathrm{~ms}$ for ytterbiumdoped fibers and is the main mechanism that depletes the upper laser level and consequently the gain between the pump pulses. This shows that the gain-switched oscillator with a pumprecovery amplifier must use an adapted pumping scheme to compensate for the losses and the different pumped conditions induced by the spontaneous emission between the laser pulses.

The compensation pumping can be realized by a low average pump power between the high pump pulses. Setting the compensation pump power to $0.9 \mathrm{~W}$ and operating the pump diodes in CW mode, the laser's peak-power dependence on the repetition rate is mitigated, as shown in Fig. 7(b) (blue circles). 

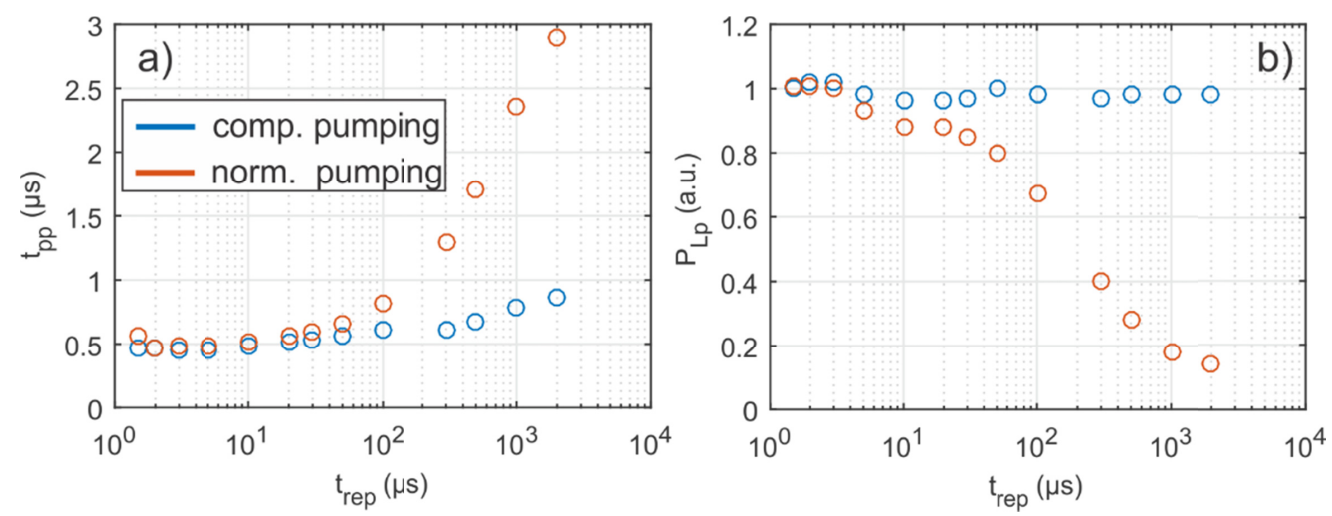

Fig. 7. Pump-pulse duration $t_{p p}$ a) and output laser peak power $\left.P_{L p} b\right)$ for the presented system plotted for different time intervals between the laser pulses. It is clear that for time intervals greater than $20 \mu$ s the depletion of the upper laser level starts to hinder the efficient operation of the laser.

The average and peak laser power achieved with the system operating at different repetition rates is shown in Fig. 8, where the measurements are plotted vs. the spacing between the laser pulses. The average achievable output laser power at the highest repetition rate of $1 \mathrm{MHz}$ was more than $30 \mathrm{~W}$. The laser peak power was kept constant for a broad range of repetition rates by the compensation pumping. In this mode the pump system was configured to produce a low $\mathrm{CW}$ power of $0.9 \mathrm{~W}$ between the high-power pump pulses to compensate for the spontaneous emission depleting the exited-ion population and producing nearly equal conditions before each high-power pump pulse.

The good result of the laser operating at different repetition rates was further tested to produce the arbitrary pulse pattern shown in Fig. 9(a). In Fig. 9(b), the same pulses are presented, while the delays in between were omitted. Both graphs are plotted for the normal pump mode, where only high-power pump pulses are used, and for the compensating mode, where the system is additionally pumped by the low pump power between them. It is clear that the normal pumping mode has a high fluctuation in the peak power from pulse to pulse, while the compensating pumping can eliminate that and achieve stability with a standard deviation of $1.5 \%$, while the stability of the pulse's FWHM was $0.7 \%$.

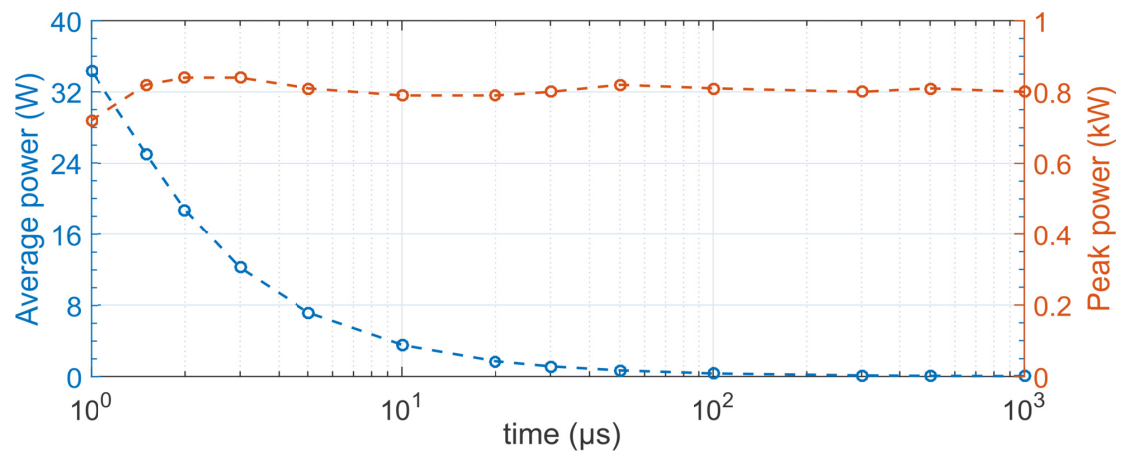

Fig. 8. Average and peak laser power shown for different time spacings between the laser pulses. 

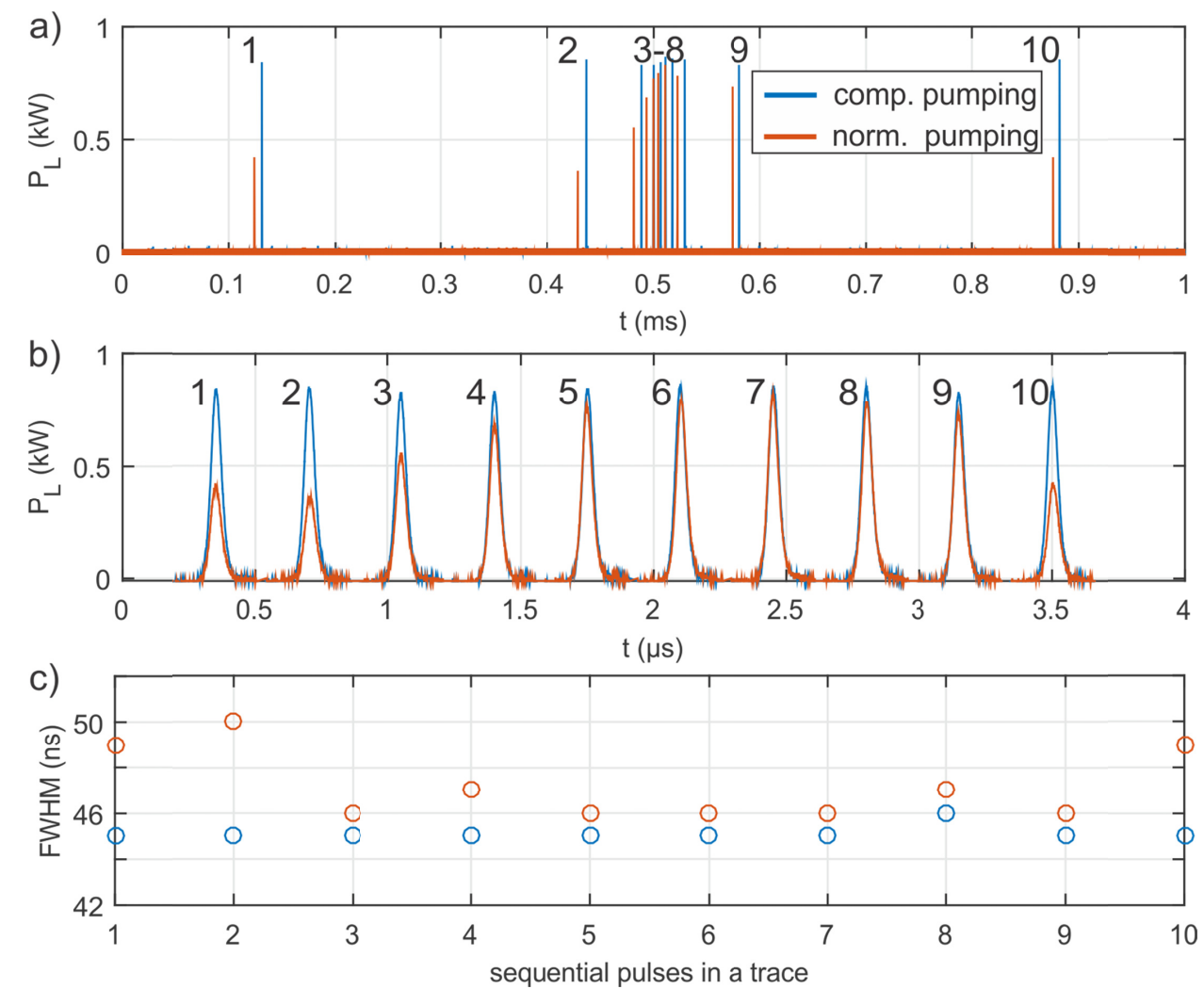

Fig. 9. Arbitrary laser-pulse pattern generated from the laser system shown in a) with the successive pulses "zoom in" shown in b) and corresponding pulse-width fluctuations shown in c). The standard deviation for the peak power in the case of compensating pumping was $1.5 \%$ and $0.7 \%$ for the FWHM.

\section{Conclusion}

The concept of a highly adaptable, gain-switched, fiber laser capable of producing laser pulses with an average power of up to $30 \mathrm{~W}$ and a peak power of approximately $1 \mathrm{~kW}$ is presented. The novel concept combines the gain-switching technique to produce pulses with the all-fiber, master-oscillator, pump-recovery amplifier for the added functionality of tunability and improved efficiency. It is ready for further power scalability to multi-kilowatt peak laser powers that can generate a pulse on demand at arbitrary times, as requested by the laser processing unit.

One advantage of the system is its ability to fine tune the laser pulse to the application, while maintaining a constant output peak power, which was previously impossible to achieve with gain-switched fiber lasers. The innovative approach of combining a fiber oscillator, a pump-recovery fiber amplifier and a tunable single-pump module enables the control of the absorbed pump power distribution through the mechanism of changing the effective absorption of the system while keeping its design complexity low.

Besides the demonstrated modes of operation made with the ytterbium-doped fibers, the same concept could be applied to gain-switched, thulium-doped fiber lasers pumped at a 793$\mathrm{nm}$ absorption peak. This peak is narrow enough to allow for the wavelength-tuning option with the change in absorption by a factor of 5 at a wavelength change of $10 \mathrm{~nm}$. This would allow the building of compact, pulsed 2- $\mu \mathrm{m}$ laser sources with the output parameters being adaptable with respect to the application. 


\section{Funding}

Slovenian Research Agency (P2-0270) (L2-8183, L2-9240).

\section{Acknowledgments}

Portions of this work were presented at the Advanced Solid-State Lasers 2018 conference in 2018 with the title A Highly Adaptable Gain Switched Fiber Laser with Improved Efficiency. The article relates to SPS Operation entitled Building blocks, tools and systems for factories of future - GOSTOP.

\section{References}

1. J. Yang, Y. Tang, and J. Xu, "Development and applications of gain-switched fiber lasers," Photon. Res. 1(1), 52-57 (2013).

2. V. Agrež, R. Petkovšek, D. Sangla, J. Saby, R. B. Picard, and F. Salin, "Effect of repetition rate on gainswitched fiber laser output pulses," Laser Phys. 24(10), 105108 (2014).

3. R. Petkovšek and V. Agrež, "Single stage Yb-doped fiber laser based on gain switching with short pulse duration," Opt. Express 22(2), 1366-1371 (2014).

4. V. Agrež and R. Petkovšek, "Gain-switched Yb-doped fiber laser for microprocessing," Appl. Opt. 52(13), 3066-3072 (2013).

5. J. Petelin, V. Agrež, B. Podobnik, and R. Petkovšek, "Short pulsed gain-switched fiber laser with improved efficiency utilizing unabsorbed pump recovery," Opt. Express 22(17), 20588-20594 (2014).

6. P. Grzes and J. Swiderski, "Gain-switched 2- $\mu \mathrm{m}$ fiber laser system providing kilowatt peak-power mode-locked resembling pulses and its application to supercontinuum generation in fluoride fibers," IEEE Photonics J. 10(1), $1-8(2018)$.

7. J. Yang, H. Li, Y. Tang, and J. Xu, "Temporal characteristics of in-band-pumped gain-switched thulium-doped fiber lasers," J. Opt. Soc. Am. B 31(1), 80-86 (2014).

8. P. F. Moulton, G. A. Rines, E. V. Slobodtchikov, K. F. Wall, G. Frith, B. Samson, and A. L. G. Carter, “Tmdoped fiber lasers: fundamentals and power scaling," IEEE J. Sel. Top. Quantum Electron. 15(1), 85-92 (2009).

9. H. Luo, F. Liu, J. Li, and Y. Liu, "High repetition rate gain-switched Ho-doped fiber laser at $2.103 \mu \mathrm{m}$ pumped by h-shaped mode-locked Tm-doped fiber laser at $1.985 \mu \mathrm{m}$," Opt. Express 26(20), 26485-26494 (2018).

10. P. Paradis, V. Fortin, Y. O. Aydin, R. Vallée, and M. Bernier, " $10 \mathrm{~W}$-level gain-switched all-fiber laser at $2.8 \mu \mathrm{m}$," Opt. Lett. 43(13), 3196-3199 (2018).

11. L. A. Zenteno, E. Snitzer, H. Po, R. Tumminelli, and F. Hakimi, "Gain switching of a $\mathrm{Nd}^{+3}$-doped fiber laser," Opt. Lett. 14(13), 671-673 (1989).

12. C. Larsen, K. P. Hansen, K. E. Mattsson, and O. Bang, "The all-fiber cladding-pumped Yb-doped gain-switched laser," Opt. Express 22(2), 1490-1499 (2014). 